\title{
ON THE ZEROS OF THE FUNCTION $\beta(z)$ ASSOCIATED WITH THE GAMMA FUNCTION*
}

\author{
BY \\ T. H. GRONWALL
}

1. Nielsen $\dagger$ has raised the question whether the function $\beta(z)$ defined by

$$
\beta(z)=\frac{1}{2}\left[\psi\left(\frac{1+z}{2}\right)-\psi\left(\frac{z}{2}\right)\right], \quad\left(\psi(z)=\frac{\Gamma^{\prime}(z)}{\Gamma(z)}\right),
$$

has any zeros, and has shown that there are no real zeros, and that the complex zeros, if any, must have their real part less than $-\frac{1}{2}$.

This question will be answered completely in the following, by showing that

The zeros of $\beta(z)$ are all complex, and their real part less than $-\frac{1}{2}$. For $n=1,2,3, \cdots$, each of the infinite strips

$$
-2 n-\frac{1}{2}<\text { real part of } z<-2 n+\frac{3}{2}
$$

contains exactly two zeros, and for $n$ sufficiently large, their asymptotic expression is

$$
\begin{aligned}
& -2 n+\frac{1}{2}+\frac{2 \log (8 n+2) \pi}{(4 n+1) \pi^{2}} \pm i \frac{\log (8 n+2) \pi}{\pi}+\frac{\xi_{n} \pm i \eta_{n}}{2 \pi n}, \\
& \xi_{n}^{2}+\eta_{n}^{2}<1 \text {. }
\end{aligned}
$$

2. From the definition of $\beta(z)$ it follows at once that

$$
\beta(z)=\sum_{n=0}^{\infty} \frac{(-1)^{n}}{z+n}=-\log 2+\frac{1}{z}+\sum_{n=1}^{\infty}(-1)^{n}\left(\frac{1}{z+n}-\frac{1}{n}\right),
$$

both series converging uniformly (and the second also absolutely) in any finite region in the $z$-plane to which the poles $z=0,-1,-2, \cdots$ are exterior; and from (1) (or directly from the definition) we obtain the relation

$$
\beta(z)+\beta(1-z)=\frac{\pi}{\sin \pi z} .
$$

* Presented to the Society, September 5, 1916; received by the editors in April, 1925.

$\dagger$ N. Nielsen, Handbuch der Theorie der Gammafunktion, Leipzig, Teubner, 1906. See p. 101. 
Let us begin by proving Nielsen's results. For $z$ real and positive, the terms in (1) have alternating signs and decrease in absolute value, so that $\beta(z)>0$. For $z=-m-\zeta$, where $m$ is a positive integer or zero, and $0<\zeta<1$, we may write (1) in the form

$$
\begin{aligned}
(-1)^{m+1} \beta(-m-\zeta)= & -\left[\frac{1}{1+\zeta}-\frac{1}{2+\zeta}+\cdots+\frac{(-1)^{m}}{m+\zeta}\right]+\frac{1}{\zeta} \\
& +\frac{1}{1-\zeta}-\left[\frac{1}{2-\zeta}-\frac{1}{3-\zeta}+\cdots\right]
\end{aligned}
$$

the terms in brackets having alternating signs and decreasing in absolute value, we have

$$
\begin{aligned}
(-1)^{m+1} \beta(-m-\zeta)>\frac{1}{\zeta}+\frac{1}{1-\zeta} & -\frac{1}{1+\zeta}-\frac{1}{2-\zeta} \\
= & \frac{1}{\zeta(1+\zeta)}+\frac{1}{(1-\zeta)(2-\zeta)}>0 .
\end{aligned}
$$

Hence there are no real zeros. On the other hand, let $z=x+y i, y \neq 0$, be a complex zero; taking the imaginary part of (1) and dividing by $y$, we find

$$
\frac{1}{x^{2}+y^{2}}-\frac{1}{(x+1)^{2}+y^{2}}+\left[\frac{1}{(x+2)^{2}+y^{2}}-\frac{1}{(x+3)^{2}+y^{2}}+\cdots\right]=0,
$$

and assuming $x>-2$, the terms in the bracket have alternating signs and decrease in absolute value, whence

$$
\frac{1}{x^{2}+y^{2}}-\frac{1}{(x+1)^{2}+y^{2}}<0 \text { or } x<-\frac{1}{2} .
$$

3. It is convenient to determine first the zeros of $\beta(1-z)$ and then replace $z$ by $1-z$. From the preceding results it follows that the zeros of $\beta(1-z)$ will be complex and have their real part greater than $\frac{3}{2}$. To obtain an asymptotic expression for $\beta(1-z)$, we observe that, for $\Re z>0(\Re z=x=$ real part of $z$ ), the expression (1) may be replaced by

$$
\beta(z)=\int_{0}^{\infty} \frac{e^{-z u}}{1+e^{-u}} d u,
$$

whence, integrating twice by parts,

$$
\beta(z)=\frac{1}{2 z}+\frac{1}{4 z^{2}}-\frac{1}{z^{2}} \int_{0}^{\infty} e^{-z u} \frac{e^{-u}-e^{-2 u}}{\left(1+e^{-u}\right)^{3}} d u .
$$


For $\Re z \geqq 1$, we have

$$
\left|\int_{0}^{\infty} e^{-u u} \frac{e^{-u}-e^{-2 u}}{\left(1+e^{-u}\right)^{3}} d u\right|<\int_{0}^{\infty} e^{-u}\left(e^{-u}-e^{-2 u}\right) d u=\frac{1}{6},
$$

and (2) and (3) now give the asymptotic expression

$$
\beta(1-z)=\frac{\pi}{\sin \pi z}-\frac{1}{2 z}-\frac{h}{z^{2}}, \quad|h|<\frac{5}{12} \text { for } \Re z \geqq 1 .
$$

4. Let us consider first the zeros of the expression

$$
\frac{\pi}{\sin \pi z}-\frac{1}{2 z}
$$

which constitutes the principal part of $\beta(1-z)$. Since $\sin \pi(x+y i)=$ $\sin \pi x \operatorname{ch} \pi y+i \cos \pi x \operatorname{sh} \pi y$, a zero $z=x+y i$ of (5) implies the two equations

$$
\begin{aligned}
& \sin \pi x \operatorname{ch} \pi y=2 \pi x, \\
& \cos \pi x \operatorname{sh} \pi y=2 \pi y,
\end{aligned}
$$

where, however, the solution $x=y=0$ must be discarded, since it corresponds to the pole $z=0$ of (5). It is evident that when $x+y i$ is a zero of (5), the same is true of $x-y i,-x+y i$ and $-x-y i$; we may therefore restrict the discussion of (6) and (7) to the case $x \geqq 0, y \geqq 0$. First assume $x=0$; since $y \geqq 0$ in this case, and ( $\operatorname{sh} \pi y) / \pi y$ increases steadily from 1 to $\infty$ as $y$ increases from 0 to $\infty,(7)$ gives $y=y_{0}$, where $y_{0}$ is the unique positive root of

$$
\frac{\operatorname{sh} \pi y_{0}}{\pi y_{0}}=2 \text {. }
$$

Now assume $x>0$; making $y=0$ in (6), we would obtain $(\sin \pi x) / \pi x=2$, whereas the quotient to the left always lies between -1 and +1 . We must consequently assume $x>0, y>0$, and (5) and (6) show that $\sin \pi x>0$, $\cos \pi x>0$, whence

$$
x=2 n+\frac{1}{2}-\xi,
$$

$n$ a positive integer or zero, $0<\xi<\frac{1}{2}$. Consider first the case $n=0$, whence $0<x<\frac{1}{2} ;$ since $\pi x / \sin \pi x<\pi / 2$ in this interval, (6) gives $\operatorname{ch} \pi y<\pi$, whence $\pi y<1.812$, and we obtain from $(7)$

$$
\cos \pi x>\frac{2 \times 1.812}{\operatorname{sh} 1.812}=1.3336>1,
$$

which is impossible. 
Next, assume $n>0$; the equations (6) and (7) become

$$
\begin{aligned}
& \cos \pi \xi \operatorname{ch} \pi y=(4 n+1) \pi-2 \pi \xi, \\
& \sin \pi \xi \operatorname{sh} \pi y=2 \pi y .
\end{aligned}
$$

Since $\pi y / \operatorname{sh} \pi y$ is a decreasing function of $y$, equation (11) represents a curve in the rectangular coördinates $\xi, y$ such that $y$ decreases steadily from $+\infty$ to $y_{0}$. when $\xi$ increases from 0 to $\frac{1}{2}$. On the curve (10), $y$ is an extremum when

$$
\cos ^{2} \pi \xi \operatorname{sh} \pi y \frac{d y}{d \xi}=[(4 n+1) \pi-2 \pi \xi] \sin \pi \xi-2 \cos \pi \xi=0,
$$

and writing this equation in the form $(4 n+1) \pi-2 \pi \xi-2 \cot \pi \xi=0$, we see that it has a unique root $\xi_{0}$ in the interval $0<\xi<\frac{1}{2}$, since the derivative of the left hand member is $2 \pi \cot ^{2} \pi \xi>0$. This root evidently corresponds to a minimum, since $y \rightarrow+\infty$ when $\xi \rightarrow \frac{1}{2}$ by (10), and we have

$$
\sin \pi \xi_{0}<\tan \pi \xi_{0}=\frac{2}{(4 n+1) \pi-2 \pi \xi_{0}}<\frac{1}{2 n \pi} .
$$

For $0<\xi<\xi_{0}$, the value of $y$ obtained from (10) decreases steadily from $y_{1}$ given by $\operatorname{ch} \pi y_{1}=(4 n+1) \pi$, to $y_{2}$, given by $\cos \pi \xi_{0} \operatorname{ch} \pi y_{2}=(4 n+1) \pi-2 \pi \xi_{0}$, while the value of $y$ obtained from (11) decreases from $+\infty$ to $y_{3}$, given by $\sin \pi \xi_{0} \operatorname{sh} \pi y_{3}=2 \pi y_{3}$. We shall now show that $y_{3}>y_{1}$; it is evidently sufficient to prove that

$$
\frac{\operatorname{sh} \pi y_{3}}{\pi y_{3}}>\frac{\operatorname{sh} \pi y_{1}}{\pi y_{1}} \text {. }
$$

From $e^{\pi y_{1}}<e^{\pi y_{1}}+e^{-\pi y_{1}}=2 \operatorname{ch} \pi y_{1}=(8 n+2) \pi$ it follows that $\pi y_{1}<\log (8 n+2) \pi$, and since $(\operatorname{sh} \pi y) / \pi y$ is a decreasing function,

$$
\frac{\operatorname{sh} \pi y_{1}}{\pi y_{1}}<\frac{\operatorname{sh}[\log (8 n+2) \pi]}{\log (8 n+2) \pi}<\frac{\frac{1}{2} e^{\log (8 n+2) \pi}}{\log (8 n+2) \pi}=\frac{(4 n+1) \pi}{\log (8 n+2) \pi} .
$$

On the other hand,

$$
\frac{\operatorname{sh} \pi y_{3}}{\pi y_{3}}=\frac{2}{\sin \pi \xi_{0}}>4 n \pi,
$$

and since we have

$$
4 n \pi>\frac{(4 n+1) \pi}{\log (8 n+2) \pi}
$$

for $n \geqq 1$, the inequality in question is established, and it follows that the two curves (10) and (11) do not intersect in the interval $0<\xi<\xi_{0}$. On the 
contrary, in the interval $\xi_{0}<\xi<\frac{1}{2}$ the $y$ in (10) increases steadily from $y_{2}$ to $+\infty$, while the $y$ in (11) decreases steadily from $y_{3}$ to $y_{0}$, and since $y_{3}>y_{1}>y_{2}$, the two curves have a unique point of intersection in the interval considered.

Returning to (5), it is thus seen that the only zeros of this function are the following:

The two zeros $y_{0} i,-y_{0} i$;

In each of the strips $2 n<\Re z<2 n+\frac{1}{2}(n=1,2,3, \cdots)$, two conjugate complex zeros;

In each of the strips $-2 n-\frac{1}{2}<\Re z<-2 n(n=1,2,3, \cdots)$, two conjugate complex zeros, equal to the preceding ones multiplied by -1 .

5. To find the distribution of the zeros of $\beta(1-z)$, we shall use the following theorem :

Let $f(z)$ and $g(z)$ be two functions, meromorphic inside a contour $C$, and holomorphic on $C$. When the inequality

$$
|f(z)-g(z)|<|g(z)|
$$

is satisfied everywhere on $C$, then neither $f(z)$ nor $g(z)$ vanishes on $C$, and the difference between the number of zeros and the number of poles of $f(z)$ inside $C$ equals the corresponding difference for $g(z) . *$

Let us apply this theorem to $f(z)=\beta(1-z)$ and $g(z)=(\pi / \sin \pi z)-(1 / 2 z)$, $C$ being the rectangle with vertices at $2 n-\frac{1}{2} \pm b i, 2 n+\frac{3}{2} \pm b i$, where $n$ is a positive integer, and $b$ positive and very large. On the horizontal sides of the rectangle, we have $z=2 n-\frac{1}{2}+x \pm b i, 0 \leqq x \leqq 2$, whence

$$
\sin \pi z=-\cos \pi x \operatorname{ch} \pi b \pm i \sin \pi x \operatorname{sh} \pi b,|\sin \pi z|^{2}=\cos ^{2} \pi x+\operatorname{sh}^{2} \pi b
$$

and

$$
\left|\frac{\pi}{\sin \pi z}\right| \leqq \frac{\pi}{\operatorname{sh} \pi b}<2 \pi e^{-\pi b} .
$$

* In the case when $C$ is a circle and $f(z)$ and $g(z)$ have no poles in its interior, this theorem is due to E. Rouché, Mémoire sur la série de Lagrange, J o u r nal d e l' E c ol e P ol y t e c h n i q u e, Cahier 39 (1862), pp. 193-224 (see Theorem III, p. 217). The theorem was rediscovered and generalized by A. Hurwitz, Ueber die Nullstellen der Bessel'schen Function, $\mathrm{M}$ a $\mathrm{t}$ h e $\mathrm{m}$ a $\mathrm{t}$ i s c h e A n$\mathrm{n}$ a l e n, vol. 33 (1889), p. 246-266 (see p. 248). Incidentally, the proof is extremely simple: First, neither $f(z)$ nor $g(z)$ vanishes on $C$, since $|f(z)-g(z)|<|g(z)|$ yields the impossible inequalities $|f(z)|$ $<0$ for $g(z)=0$ and $|g(z)|<|g(z)|$ for $f(z)=0$. In the identity

$$
\log f(z)=\log g(z)+\log [1+(f(z)-g(z)) g(z)]
$$

we perform the analytic continuation of both members along the contour $C$, described once in the positive sense. Then $\log f(z)$ will increase by $2 \pi i$ times the difference between the number of zeros and the number of poles of $f(z)$ interior to $C$, the increase in $\log g(z)$ will be the corresponding expression for $g(z)$, and the third logarithm does not change, since, on account of the inequality $|f(z)-g(z)|<$ $|g(z)|$, the point $\zeta=[f(z)-g(z)] g(z)$ describes a closed path interior to the circle $|\zeta|<1$ where $\log (1+\zeta)$ is holomorphic. 
Moreover, for $b>2 n+2$,

$$
\frac{1}{2|z|}>\frac{1}{4 n-1+2 x+2 b}>\frac{1}{4 b}, \frac{1}{|z|^{2}}=\frac{1}{\left(2 n-\frac{1}{2}+x\right)^{2}+b^{2}}<\frac{1}{b^{2}},
$$

whence, for $b$ sufficiently large and using (4),

$$
\begin{aligned}
&\left|\frac{\pi}{\sin \pi z}-\frac{1}{2 z}\right| \geqq \frac{1}{2|z|}-\left|\frac{\pi}{\sin \pi z}\right|>\frac{1}{4 b}-2 \pi e^{-\pi b}, \\
&\left|\beta(1-z)-\left(\frac{\pi}{\sin \pi z}-\frac{1}{2 z}\right)\right|<\frac{5}{12|z|^{2}}<\frac{5}{12 b^{2}}<\frac{1}{4 b}-2 \pi e^{-\pi b} \\
&<\left|\frac{\pi}{\sin \pi z}-\frac{1}{2 z}\right| .
\end{aligned}
$$

On the vertical sides of the rectangle, we have $z=2 m-\frac{1}{2}+y i, m=n$ or $n+1$ and $-b \leqq y \leqq b$, whence $\sin \pi z=-\operatorname{ch} \pi y$ and

$$
\frac{\pi}{\sin \pi z}-\frac{1}{2 z}=-\left(\frac{\pi}{\operatorname{ch} \pi y}+\frac{1}{4 m-1+2 y i}\right) ;
$$

the real part of the second term to the right having the same sign as the first term, it follows that

$$
\left|\frac{\pi}{\sin \pi z}-\frac{1}{2 z}\right|>\left|\frac{1}{4 m-1+2 y i}\right|=\frac{1}{|2 z|} .
$$

Hence we see that

$$
\left|\beta(1-z)-\left(\frac{\pi}{\sin \pi z}-\frac{1}{2 z}\right)\right|<\frac{5}{12|z|^{2}}<\frac{1}{2|z|}<\left|\frac{\pi}{\sin \pi z}-\frac{1}{2 z}\right| .
$$

For $b$ sufficiently large, the conditions of the theorem are this fulfilled. According to paragraph 4, the function $(\pi / \sin \pi z)-(1 / 2 z)$ has two zeros in our rectangle for $b$ sufficiently large, viz., those with their real part between $2 n$ and $2 n+\frac{1}{2}$, and on the other hand, there are the two poles $2 n$ and $2 n+1$. The function $\beta(1-z)$ has the same poles in the rectangle, and our theorem therefore shows that the rectangle contains exactly two zeros of $\beta(1-z)$. Letting $b$ increase indefinitely, and replacing $z$ by $1-z$, it is thus seen that for $n=1,2,3, \cdots$, each strip $-2 n-\frac{1}{2}<\Re z<-2 n+\frac{3}{2}$ contains two zeros of $\beta(z)$.

6. To obtain asymptotic expressions for the zeros, we begin with those of $(\pi / \sin \pi z)-(1 / 2 z)$. As in paragraph 4 , it is sufficient to consider the case $x>0, y>0$, and it follows from (10) that $\operatorname{ch} \pi y>4 n \pi$, so that $y$ increases 
indefinitely with $n$, and formula (11) shows that then $\lim \sin \pi \xi=0$, whence $\lim \cos \pi \xi=1$. By (10), we now have

$$
\begin{gathered}
\lim \frac{\operatorname{ch} \pi y}{(4 n+1) \pi}=1=\lim \frac{e^{\pi y}}{2(4 n+1) \pi}, \\
\pi y=\log [(8 n+2) \pi(1+\eta)], \quad \lim \eta=0 .
\end{gathered}
$$

Substituting this value of $\pi y$ in (11), it is seen that

$$
\begin{aligned}
\lim \frac{(4 n+1) \pi}{2 \log (8 n+2) \pi} \cdot \pi \xi= & \lim \frac{\pi \xi}{\sin \pi \xi} \cdot \lim \frac{(4 n+1) \pi}{2 \log (8 n+2) \pi} \\
& \cdot \frac{2 \log [(8 n+2) \pi(1+\eta)]}{\frac{1}{2}\left[(8 n+2) \pi(1+\eta)-\frac{1}{(8 n+2) \pi(1+\eta)}\right]} \\
= & 1, \quad \lim \xi^{\prime}=0 . \\
\pi \xi & =\frac{2 \log (8 n+2) \pi}{(4 n+1) \pi}\left(1+\xi^{\prime}\right),
\end{aligned}
$$

Denoting, as usual, by $f(x)=O(g(x))$ the fact that two constants $A$ and $x_{0}$ exist such that $|f(x)|<A g(x)$ for $x>x_{0}$, we find by substituting the above values of $\pi y$ and $\pi \xi$ in (10) and expanding $\cos \pi \xi$ in a power series, so that $\cos \pi \xi=1+O\left(\xi^{2}\right)=1+O((\log n) / n)^{2}$,

$$
\begin{aligned}
{\left[1+O\left(\left(\frac{\log n}{n}\right)^{2}\right)\right] } & {\left[(4 n+1) \pi(1+\eta)-\frac{1}{4(4 n+1) \pi(1+\eta)}\right] } \\
= & (4 n+1) \pi+O\left(\frac{\log n}{n}\right),
\end{aligned}
$$

and it follows that

$$
\eta=O\left(\left(\frac{\log n}{n}\right)^{2}\right) .
$$

Finally (11) gives, taking account of the order of magnitude of $\eta$,

$$
\begin{gathered}
{\left[\frac{2 \log (8 n+2) \pi}{(4 n+1) \pi}\left(1+\xi^{\prime}\right)+O\left(\left(\frac{\log n}{n}\right)^{3}\right)\right]\{(4 n+1) \pi[1} \\
\left.\left.+O\left(\left(\frac{\log n}{n}\right)^{2}\right)\right]-\frac{1}{4(4 n+1) \pi}\left[1+O\left(\left(\frac{\log n}{n}\right)^{2}\right)\right]\right\} \\
=2\left[\log (8 n+2) \pi+O\left(\left(\frac{\log n}{n}\right)^{2}\right)\right], \\
2 \log (8 n+2) \pi \cdot \xi^{\prime}=O\left(\frac{(\log n)^{3}}{n^{2}}\right),
\end{gathered}
$$


whence

$$
\xi^{\prime}=O\left(\left(\frac{\log n}{n}\right)^{2}\right)
$$

For the two zeros of $(5)$ in the strip $2 n<\Re z<2 n+\frac{1}{2}$, we consequently have the asymptotic expression

$$
2 n+\frac{1}{2}-\frac{2 \log (8 n+2) \pi}{(4 n+1) \pi^{2}} \pm i \frac{\log (8 n+2) \pi}{\pi}+O\left(\left(\frac{\log n}{n}\right)^{2}\right)
$$

7. We shall now prove that for $n$ sufficiently large, $\beta(1-z)$ has one zero in the neighborhood of each of the zeros (12) of its principal part (5). Consider the circle

$$
\begin{gathered}
z=2 n+\frac{1}{2}-\frac{2 \log (8 n+2) \pi}{(4 n+1) \pi^{2}}+i \frac{\log (8 n+2) \pi}{\pi}+\frac{\xi+\eta i}{2 n \pi}, \\
\xi^{2}+\eta^{2}<1 ;
\end{gathered}
$$

by (12), this circle contains in its interior a single zero of (5) when $n$ is sufficiently large. On the circumference $\xi^{2}+\eta^{2}=1$, we have

$$
\begin{aligned}
\sin \pi z= & \frac{1}{2 i}\left[\frac{i}{(8 n+2) \pi} e^{-\frac{2 i \log (8 n+2) \pi}{(4 n+1) \pi}+\frac{\xi i-\eta}{2 n}}\right. \\
& +i(8 n+2) \pi e^{\left.\frac{2 i \log (8 n+2) \pi}{(4 n+1) \pi}+\frac{\eta-\xi i}{2 n}\right]} \\
= & (4 n+1) \pi\left[1+\frac{2 i \log (8 n+2) \pi}{(4 n+1) \pi}+\frac{\eta-\xi i}{2 n}+O\left(\left(\frac{\log n}{n}\right)^{2}\right)\right], \\
\frac{\pi}{\sin \pi z} & =\frac{1}{4 n+1}\left[1-\frac{2 i \log (8 n+2) \pi}{(4 n+1) \pi}+\frac{\xi i-\eta}{2 n}+O\left(\left(\frac{\log n}{n}\right)^{2}\right)\right], \\
\frac{1}{2 z} & =\frac{(4 n+1)\left[1+\frac{2 i \log (8 n+2) \pi}{(4 n+1) \pi}+O\left(\frac{\log n}{n^{2}}\right)\right]}{(1)}\left[1-\frac{2 i \log (8 n+2) \pi}{(4 n+1) \pi}+O\left(\frac{\log n}{n^{2}}\right)\right],
\end{aligned}
$$

whence

$$
\frac{\pi}{\sin \pi z}-\frac{1}{2 z}=\frac{\xi i-\eta}{2 n(4 n+1)}+O\left(\frac{(\log n)^{2}}{n^{3}}\right)=\frac{\xi i-\eta}{8 n^{2}}+O\left(\frac{(\log n)^{2}}{n^{3}}\right),
$$


and since $\xi^{2}+\eta^{2}=1$,

$$
\left|\frac{\pi}{\sin \pi z}-\frac{1}{2 z}\right|=\frac{1}{8 n^{2}}+O\left(\frac{(\log n)^{2}}{n^{3}}\right) .
$$

On the other hand, we have on the same circumference, according to (4),

$$
\left|\beta(1-z)-\left(\frac{\pi}{\sin \pi z}-\frac{1}{2 z}\right)\right|<\frac{5}{12|z|^{2}}=\frac{5}{48 n^{2}}+O\left(\frac{\log n}{n^{3}}\right),
$$

and by comparison with (14)

$$
\left|\beta(1-z)-\left(\frac{\pi}{\sin \pi z}-\frac{1}{2 z}\right)\right|<\left|\frac{\pi}{\sin \pi z}-\frac{1}{2 z}\right|
$$

on the circumference $\xi^{2}+\eta^{2}=1$ for $n$ sufficiently large. Applying the theorem in paragraph 5 , we see that the circle $\xi^{2}+\eta^{2}<1$ contains a single zero of $\beta(1-z)$, and the same is evidently true of the circle obtained by changing the sign of $i$ in (13). We have thus obtained asymptotic expressions for the two zeros of $\beta(1-z)$ contained in the strip $2 n-\frac{1}{2}<\Re z<2 n+\frac{3}{2}$, and replacing $z$ by $1-z$, we finally arrive at the result stated in paragraph 1 .

It is clear that closer approximations to the zeros may be obtained by using, instead of (5), the general asymptotic expansion

$$
\begin{aligned}
\beta(z)=\frac{1}{2 z} & +\sum_{\nu=1}^{n}(-1)^{\nu-1} \frac{2^{2 \nu}-1}{2 \nu} B_{\nu} \cdot \frac{1}{z^{2 \nu}} \\
& +\frac{1}{z^{2 n}} \int_{0}^{\infty} e^{-z u} \frac{d^{n}}{d u^{n}}\left(\frac{1}{1+e^{-u}}\right) d u,
\end{aligned}
$$

valid for $\Re z>0$, where $B$, are the Bernoulli numbers.

NEW York, N. Y. 